\title{
Características biológicas e comportamentais de Neodohrniphora elongata Brown (Diptera, Phoridae), um parasitóide da saúva Atta sexdens rubropilosa Forel (Hymenoptera, Formicidae)
}

\author{
Marcos A. L. Bragança ${ }^{1}$, Athayde Tonhasca $\mathrm{Jr}^{2}{ }^{2}$ Terezinha M. C. Della Lucia ${ }^{3}$
}

${ }^{1}$ Curso de Ciências Biológicas, Campus de Porto Nacional, Universidade Federal do Tocantins, 77500-000 Porto Nacional-TO, Brasil. marcosbr@uft.edu.br

${ }^{2}$ Scottish Natural Heritage, Great Glen House, Inverness, IV3 8NW, Reino Unido. athayde.tonhasca@snh.gov.uk

${ }^{3}$ Departamento de Biologia Animal, Universidade Federal de Viçosa, 36570-000 Viçosa-MG, Brasil. tdlucia@ufv.br

\begin{abstract}
Biological and behavioral characteristics of Neodohrniphora elongata Brown (Diptera, Phoridae), a parasitic fly of the leaf-cutting ant Atta sexdens rubropilosa Forel (Hymenoptera, Formicidae). Characteristics of the biology and behavior of the phorid Neodohrniphora elongata Brown, 2001 in relation to workers of the host Atta sexdens rubropilosa Forel, 1908 were studied. Twenty-four field-collected females of $N$. elongata were released singly inside an observation chamber placed between a nest of A. sexdens rubropilosa and a foraging arena. Flies launched attacks on flies and failed four or five times before they would successfully attack ants and oviposit on the head of workers. Larvae of the parasitoid developed in the cephalic capsule of $63.8 \%$ of the 426 attacked workers; 218 flies emerged. $N$. elongata oviposited on bigger workers, or those with cephalic capsules $2.9 \pm 0.4 \mathrm{~mm}$ wide. Capsule width seems to be important for the development of the parasitoid because failure in pupa formation and the non-emergence of adults occurred primarily among ants with capsules smaller than $2.9 \mathrm{~mm}$. Flies that emerged in the laboratory had greater longevity when fed on honey solution at $10 \%$ than honey solution at $50 \%$, or on distilled water only. The females emerged in laboratory had flight and attack behavior similar to those of field females, although second generation parasitoids could not be obtained. Additional studies on the impact of natural sources of carbohydrates and protein on the longevity and reproduction of $N$. elongate should be conducted to multiply this and other phorids of leaf-cutting ants in the laboratory.
\end{abstract}

KEYWORDS. Biological control; fertility; longevity; parasitism.

RESUMO. Características biológicas e comportamentais de Neodohrniphora elongata Brown (Diptera, Phoridae), um parasitóide da saúva Atta sexdens rubropilosa Forel (Hymenoptera, Formicidae). Foram investigadas as características da biologia e comportamento do forídeo Neodohrniphora elongata Brown, 2001 em relação às operárias do hospedeiro Atta sexdens rubropilosa Forel, 1908. Vinte e quatro fêmeas de $N$. elongata coletadas no campo foram liberadas, uma por vez, em uma cuba de observação interposta entre um ninho de A. sexdens rubropilosa e uma arena de forrageamento. As moscas realizaram de quatro a cinco vezes mais investidas sem sucesso contra as formigas do que ataques efetivos, quando elas ovipositaram na cabeça das operárias. Houve ataques em 426 operárias e desenvolvimento da larva do parasitóide na cápsula cefálica de 63,8\% delas, sendo que emergiram 218 moscas. $N$. elongata ovipositou nas maiores operárias, ou seja, naquelas com largura da cápsula cefálica de $2,9 \pm 0,4 \mathrm{~mm}$, o que parece ser importante para o desenvolvimento do parasitóide, pois o fracasso na formação de pupas ou a não emergência do adulto ocorreram principalmente em formigas com largura da cápsula inferior a $2,9 \mathrm{~mm}$. As moscas que emergiram em laboratório tiveram maior longevidade quando alimentadas com solução de mel $10 \%$ do que com solução de mel $50 \%$ ou somente água destilada. As fêmeas que emergiram no laboratório exibiram os mesmos comportamentos de voo e ataque das fêmeas do campo, mas não foi possível obter parasitóides de segunda geração. Estudos adicionais devem ser realizados para investigar a adequação de fontes naturais de carboidratos e proteínas sobre a longevidade e reprodução de $N$. elongata, visando à multiplicação deste e de outros forídeos de saúvas em laboratório.

PALAVRAS-CHAVE. Controle biológico; fertilidade; longevidade; parasitismo.

As formigas cortadeiras dos gêneros Atta Fabricius, 1804 e Acromyrmex Mayr, 1865 são consideradas insetos polífagos comuns em florestas primárias da maior parte da América do Sul. Algumas espécies destas formigas constantemente invadem áreas de agricultura e de florestas implantadas, onde se tornam pragas (Fowler et al. 1989; Cherrett 1986). A utilização de substâncias químicas em iscas é a principal forma de controle das espécies pragas, mas também tem sido buscado o aprimoramento dos programas de manejo, inclusive com a utilização de técnicas que beneficiem o seu controle biológico natural (Zanetti et al. 2000; Antunes et al. 2000, 2005 ). Essas formigas possuem diversos inimigos naturais, entre eles as moscas parasitóides da família Phoridae (Feener \& Moss 1990; Brown 2001). Weber (1972) sugeriu que forídeos parasitóides poderiam ser úteis em diminuir as populações das formigas cortadeiras.

As espécies de forídeos parasitóides de saúvas pertencem a três gêneros: Apocephalus Coquillett, 1901, Myrmosicarius Borgmeier, 1928 e Neodohrniphora Malloch, 1914 (Borgmeier 1928, 1931; Disney \& Bragança 2000; Brown 2001). Embora 
causem baixos índices de parasitismo natural em saúvas ( 2 a $4 \%$ ), os forídeos são capazes de alterar negativamente o comportamento de forrageamento de seus hospedeiros, principalmente pela diminuição do número e tamanho das forrageadoras ao longo das trilhas e da massa de fragmentos vegetais transportados para o ninho (Orr 1992; Bragança et al. 1998; Tonhasca et al. 2001). Esses parasitóides podem coexistir em uma mesma trilha de forrageamento de Atta spp., selecionando operárias de diferentes classes de tamanho para atacar e, consequentemente, podem ter um efeito combinado na redução do forrageamento (Tonhasca et al. 2001; Bragança $\&$ Medeiros 2006).

Durante os ataques, as fêmeas de Neodohrniphora sobrevoam as operárias de Atta em suas trilhas e ovipositam na cabeça ou abdome do hospedeiro, o que desencadeia comportamentos de defesa específicos das saúvas. Uma única larva alimenta-se do conteúdo da cápsula cefálica da formiga, onde completa seu desenvolvimento antes de empupar entre as mandíbulas, mesmo que a oviposição seja realizada no abdome do hospedeiro (Feener \& Brown 1993; Tonhasca 1996; Bragança et al. 2002, 2003). Alguns destes estudos mostram que a simulação das condições de campo em laboratório permite o comportamento de ataque de fêmeas de Neodohrniphora spp. coletadas em campo e a obtenção de formigas parasitadas, que resultam na emergência dos parasitóides.

As informações sobre os efeitos negativos de Neodohrniphora spp. no forrageamento de saúvas e a possibilidade deles completarem seu desenvolvimento em laboratório indicam o potencial desses parasitóides como agentes de controle biológico. Porém, para a multiplicação destes forídeos em laboratório e sua posterior liberação no campo, de modo semelhante ao que foi realizado com forídeos do gênero Pseudacteon Coquillett, 1907, parasitóides de formigas do gênero Solenopsis Westwood, 1840 (Porter et al. 1997; Morrison \& Porter 2005; Vazquez et al. 2006), há a necessidade de ampliar os conhecimentos sobre seus aspectos biológicos e comportamentais. Algumas das informações essenciais ainda desconhecidas sobre as interações entre saúvas e forídeos são sobre a alimentação, longevidade e fertilidade dessas moscas em laboratório. Da mesma forma, não é conhecido se as castas reprodutivas de saúvas também são parasitadas por forídeos.

Neodohrniphora elongata Brown, 2001 é um forídeo parasitóide específico de Atta sexdens rubropilosa Forel, 1908 (Brown 2001), uma das saúvas economicamente mais importantes no Brasil. Apesar de este parasitóide ser comum em trilhas de seu hospedeiro na região Sudeste do Brasil, ainda não existe informações sobre sua biologia e comportamento. Os objetivos deste trabalho foram avaliar os aspectos biológicos e comportamentais básicos de $N$. elongata como um parasitóide de A. sexdens rubropilosa, investigando também a longevidade e fertilidade das moscas sob diferentes condições de alimentação. Também foi avaliada a possibilidade de parasitismo de iças desse hospedeiro pelo forídeo $N$. elongata.

\section{MATERIALE MÉTODOS}

Ataques de N. elongata e obtenção do parasitóide em laboratório.

Dezoito fêmeas de N. elongata foram coletadas enquanto perseguiam operárias de $A$. sexdens rubropilosa ao longo de trilhas de quatro formigueiros, localizados em uma área de mata no campus da Universidade Federal de Viçosa, Viçosa ( $\left.20^{\circ} 45^{\prime} \mathrm{S}, 42^{\circ} 51^{\prime} \mathrm{O}\right)$, Minas Gerais, Brasil. Os parasitóides foram mantidos em laboratório e alimentados com chumaço de algodão embebido com solução aquosa de mel $10 \%$ por até 12 horas antes de serem liberados, individualmente, em uma cuba de observação em acrílico ( $100 \mathrm{~cm}$ de comprimento, $45 \mathrm{~cm}$ de largura e $40 \mathrm{~cm}$ de altura), conectada por mangueiras plásticas ( $35 \mathrm{~cm}$ de comprimento e $2 \mathrm{~cm}$ de diâmetro) a um ninho de $A$. sexdens rubropilosa de 3,5 anos de idade e uma arena de forrageamento. A cuba tinha duas aberturas frontais de $10 \mathrm{~cm}$ de diâmetro com tampas circulares, também de acrílico, e com o fundo e as laterais substituídos por tecido organza branco para permitir a circulação de ar. Temperatura, umidade e fotoperíodo no laboratório foram mantidos a $23 \pm 1^{\circ} \mathrm{C}, 80 \pm 5 \%$ UR e 10 horas de luz, respectivamente.

Os números de toques e de ataques de cada mosca contra as formigas foram registrados durante 60 minutos. Em um mesmo dia, foram liberadas até duas moscas na cuba. $\mathrm{O}$ ataque de $N$. elongata para ovipositar em operárias de A. sexdens rubropilosa foi considerado como o contato da mosca com a parte posterior da cabeça da formiga por pelo menos 1 segundo, enquanto que um toque correspondeu à investida em que a mosca apenas tocava qualquer parte do corpo da formiga por menos de um segundo (Tonhasca et al. 2001). As moscas que não iniciaram voos de perseguição às formigas, realizando toques e ataques, até cinco minutos após liberação na cuba foram descartadas. Imediatamente antes da liberação de cada mosca na cuba, o fluxo de formigas que retornavam para o ninho foi medido em cinco sessões consecutivas de três minutos, na distância média da mangueira que conectava o ninho à cuba, e correlacionado com o número de toques e de ataques. Como as espécies de forídeos do gênero Neodohrniphora preferem, em geral, realizar ataques contra as maiores operárias da colônia (Tonhasca 1996; Bragança et al. 2002), e as características do substrato vegetal podem afetar o tamanho médio das operárias e a intensidade do fluxo de operárias (Tonhasca \& Bragança 2000; Silva et al. 2007), a colônia foi alimentada com folhas de uma única espécie de planta (Acalypha wilkesiana Müll. Arg., 1866, var. hoffmanii, Euphorbiaceae).

As formigas atacadas pelas moscas foram retiradas da cuba com uma pinça, marcadas com coloração específica no tórax ou abdome com tinta sem odor e não tóxica $\left(\right.$ Painters $\left.^{\circledR}\right)$ e recolocadas na cuba após 10 a 15 minutos. Desta forma, foi possível registrar também a recorrência de ataques em uma formiga por uma mesma fêmea ou por fêmea de N. elongata diferente.

Após a liberação das 18 moscas na cuba, outras 20 fêmeas do parasitóide $N$. elongata foram coletadas e os procedimentos 


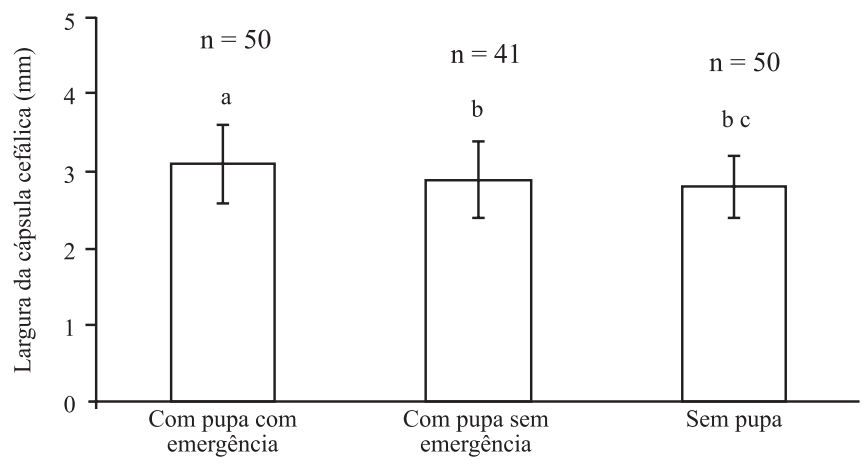

Fig. 1. Comparação da largura da cápsula cefálica (média \pm desvio padrão) de operárias de $A$. sexdens rubropilosa atacadas por $N$. elongata que tiveram formação da pupa do parasitóide (com e sem emergência da mosca) e sem formação da pupa (ANOVA: $\mathrm{F}_{2,138}=4,24 ; \mathrm{P}=0,016$ ). Médias seguidas da mesma letra não diferem pelo teste da Diferença Mínima Significativa (DMS).

em laboratório descritos acima foram repetidos para um segundo ninho de $A$. sexdens rubropilos a de idade semelhante ao primeiro, mas com maior atividade em termos de fluxo de formigas.

Nos dias subseqüentes às liberações dos forídeos na cuba, a pilha de resíduos produzidos pela colônia ("lixo") era examinada diariamente para recolhimento das formigas mortas com marca de tinta, que eram colocadas em placas de Petri e levadas a uma câmara climatizada $\left(26,5 \pm 0,5^{\circ} \mathrm{C}, 85 \pm 5 \%\right.$ UR e ausência de luz) por 24 horas. Após esse período, as formigas parasitadas eram transferidas individualmente para tubos de vidro (8,2 x 2,3 cm), que eram tampados com algodão e mantidos na câmara por 40 dias para registrar-se a emergência das moscas. Uma amostra de $20 \%$ das formigas marcadas recuperadas do lixo e que não apresentavam sinais externos de parasitismo, ou seja, a pupa do parasitóide entre as mandíbulas da formiga (Tonhasca 1996), foi dissecada para a detecção de larvas do forídeo.

O tamanho médio das forrageadoras atacadas pelo parasitóide foi comparado com o tamanho médio de uma amostra ao acaso das forrageadoras que transitavam na cuba imediatamente antes da liberação da mosca. Também foram feitas comparações do tamanho médio entre as operárias atacadas sem formação de pupa do parasitóide, com pupa mas sem emergência do parasitóide e com emergência da mosca. A maior largura da cápsula cefálica, medida com um microscópio estereoscópico provido de ocular micrométrica $(0,1 \mathrm{~mm}$ de precisão), foi utilizada como indicação do tamanho das formigas (Wilson 1980).

Uma parte das moscas coletadas em campo e obtidas em laboratório foi enviada ao Dr. Brian V. Brown (Natural History Museum of Los Angeles County) para identificação e a outra parte foi depositada na coleção de Insetos do Centro de Ciências e Tecnologias Agropecuárias da Universidade Estadual do Norte Fluminense, em Campos dos Goytacazes, Rio de Janeiro.
Longevidade de $N$. elongata.

Os forídeos machos e fêmeas que emergiram das operárias do segundo ninho foram mantidos individualmente nos mesmos tubos e na câmara climatizada sob quatro diferentes condições de alimentação para a comparação da longevidade: solução aquosa de mel 10\%, solução aquosa de mel 50\%, água destilada e jejum. As soluções de mel e a água foram oferecidas em algodão embebido, o qual foi trocado diariamente até a morte dos parasitóides. A sexagem das moscas foi realizada com base na morfologia da terminália.

Fertilidade de $N$. elongata.

Fêmeas do parasitóide que emergiram de operárias do segundo ninho foram liberadas, individualmente, na cuba acoplada a um terceiro ninho de $A$. sexens rubropilosa, com o objetivo de se obter formigas parasitadas e avaliar a fertilidade de fêmeas de $N$. elongata que emergiram em laboratório. Essas fêmeas, com idade de um a 10 dias, foram utilizadas em testes sob os seguintes tratamentos: (i) jejum, (ii) alimentadas com solução aquosa de mel 10\%, (iii) alimentadas com solução aquosa de mel $50 \%$, (iv) alimentadas e com contato prévio com machos por 24 a $48 \mathrm{~h}$ dentro do tubo e (v) alimentadas e acompanhadas de machos, mas sem contato prévio entre eles. Todos os machos utilizados foram alimentados. O comportamento de ataque e o número de ataques de cada fêmea foram registrados por até três horas consecutivas. Após este período, algumas fêmeas que ainda estavam realizando ataques foram deixadas na cuba por mais algumas horas sem registro do número de ataques. Nesta etapa, as formigas atacadas não receberam marcação e a maioria permaneceu na colônia, com exceção de uma amostra de cada tratamento que foi retirada da cuba após cada ataque, dividida em grupos de cinco indivíduos por placa de Petri e mantida na câmara climatizada por 20 dias para observação de possíveis sinais de parasitismo. Estas últimas foram alimentadas com solução de mel 10\%. Ao mesmo tempo, por quinze dias após o encerramento dos testes, todas as formigas mortas com um tamanho igual ou superior as 1,7 $\mathrm{mm}$ eram removidas do lixo, diariamente, e levadas para a câmara por 24 horas para a detecção de indivíduos com pupa de $N$. elongata. A seleção das formigas removidas do lixo foi feita subjetivamente, baseando-se em estimativas visuais resultantes de medições de formigas atacadas que foram marcadas nos testes anteriores, quando foi determinado que $1,7 \mathrm{~mm}$ era o menor tamanho da cápsula cefálica de operárias de A. sexdens rubropilosa atacadas pelo parasitóide em laboratório.

Ataques de $N$. elongata a içás de A. sexdens rubropilosa.

Em período de revoada de $A$. sexdens rubropilosa na mesma área em estudo, foram feitas observações em campo e laboratório para avaliar o comportamento e possível parasitismo de $N$. elongata em relação à casta permanente fêmea antes do voo nupcial (içás). As observações de campo foram feitas em olheiros de dois ninhos em dois períodos de tempo de aproximadamente 40 minutos cada, quando foi registrado o comportamento dos forídeos que se encontravam 
próximos a esses olheiros. Em seguida, foram coletadas cinco fêmeas do parasitóide que estavam realizando ataques às operárias em um dos ninhos e 50 içás. Em laboratório, um forídeo e dez içás (cinco com asas e cinco que tiveram as asas removidas) foram liberados na cuba de observação, que foi desconectada do ninho e da arena de forrageamento, para registro do comportamento da mosca por 60 minutos. Foram realizadas cinco liberações de um forídeo e dez içás.

Análise de dados.

$\mathrm{O}$ teste $\mathrm{t}$ foi utilizado para comparações entre ninhos quanto ao fluxo de formigas, ao número de toques e de ataques dos forídeos e à relação número de toques por ataque. $\mathrm{O}$ teste $\mathrm{t}$ foi utilizado também para comparar o tamanho de duas amostras de formigas ou de moscas. O tamanho de 30 fêmeas de $N$. elongata coletadas ao acaso no campo foi comparado com o de fêmeas emergidas em laboratório, sendo que o comprimento da asa foi utilizado como indicador do tamanho do forídeo (Morrison et al. 1997). A análise de regressão linear foi utilizada para avaliar o efeito do fluxo de formigas sobre o número de toques e de ataques realizados pelos forídeos. Os dados de longevidade dos parasitóides mantidos sob diferentes regimes alimentares e da largura da cápsula cefálica das formigas atacadas (sem formação de pupa, com pupa malograda e com emergência da mosca) foram submetidos à análise de variância, e a separação de médias foi feita pelo teste da Diferença Mínima Significativa (DMS). Os dados de longevidade, fluxo de formigas, número de toques e de ataques e relação do número de toques por ataque foram transformados para $\log$ (x) para homogeneizar as variâncias. Nos resultados, as médias são seguidas de desvios padrões.

\section{RESULTADOS}

Das 38 moscas coletadas no campo e liberadas na cuba de observação, nove no primeiro ninho e 15 no segundo iniciaram perseguição às formigas imediatamente ou até cinco minutos após liberação. Elas investiram contra qualquer formiga em trânsito na cuba, tocando qualquer parte de seu corpo, mas os ataques ocorreram principalmente na parte posterior da cabeça das maiores operárias sem carga. Formigas imóveis ou com carga raramente foram atacadas. Não houve diferença significativa na relação do número de toques por ataque entre os ninhos, mas o fluxo médio de formigas, o número de toques e o número de ataques no segundo formigueiro foram maiores do que no primeiro (Tabela I). Não houve correlação dos números de toques e de ataques com o fluxo médio de formigas de cada um dos dois ninhos. O tamanho médio das forrageadoras atacadas pelo parasitóide $(2,9 \pm 0,4 \mathrm{~mm} ; \mathrm{n}=$ $250)$ foi significativamente maior $(\mathrm{t}=22,56 ; \mathrm{P}<0,0001)$ do que o tamanho de forrageadoras em trânsito na cuba $(2,3 \pm 0,4 \mathrm{~mm}$; $\mathrm{n}=200$ ) e que, portanto, estavam sujeitas ao ataque das moscas. Estas medidas foram unificadas para os dois ninhos porque não houve diferença significativa entre estes.

No momento do ataque, $N$. elongata aproxima-se pela frente ou lados e posiciona-se sobre a cabeça do hospedeiro, em

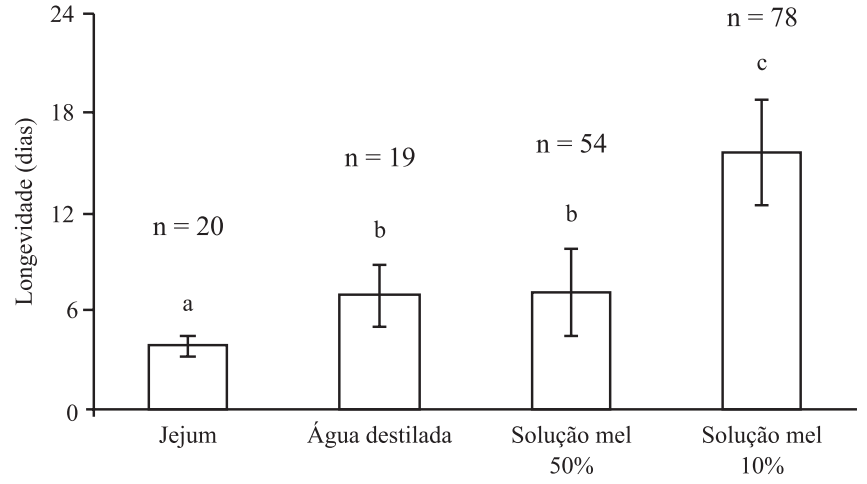

Fig. 2. Comparação da longevidade (média \pm desvio padrão) de adultos de $N$. elongata que emergiram em laboratório sob diferentes condições de alimentação (ANOVA: $\mathrm{F}_{3 ; 167}=162,12 ; \mathrm{P}<0,001$ ). Médias seguidas da mesma letra não diferem pelo teste da Diferença Mínima Significativa (DMS).

sentido contrário ao da formiga, aparentemente introduzindo o ovipositor na extremidade posterodorsal da cabeça da operária. O comportamento de defesa exibido pelas operárias de A. sexdens rubropilosa foi caracterizado pelo hospedeiro fugindo ou atacando o parasitóide com as mandíbulas ou abaixando-se e posicionando as pernas sobre sua cabeça para evitar o ataque da mosca. Após o ataque, a formiga permanecia imóvel e era imediatamente cercada por 15 a 25 companheiras, que tocavam sucessivamente a operária recém-atacada com as antenas e peças bucais por três a cinco min antes de dispersarem.

A larva de $N$. elongata se alimentou do conteúdo da cápsula cefálica e levou o hospedeiro à morte. Após a morte, as formigas parasitadas, identificadas pela marcação, foram depositadas pelas companheiras na pilha de resíduos da colônia, juntamente com as operárias não parasitadas. Os indivíduos parasitados eram identificados pela presença de uma pupa do parasitóide entre suas mandíbulas e por apresentarem as partes do corpo e do aparelho bucal coalescentes. A parte exposta da pupa, cerca de um terço do seu comprimento, apresentava coloração semelhante ao do corpo da formiga e dois sifões respiratórios mais escuros. O período desde a oviposição até o surgimento da pupa entre as mandíbulas, que corresponde ao período de incubação dos ovos mais o período larval, e o período pupal foram $9,1 \pm 1,2$ dias $(n=120)$ e $24,5 \pm 1,2$ dias $(n=120)$, respectivamente.

Apesar de ter sido registrado um total de 494 ataques, 426 operárias foram marcadas com tinta, pois $68(13,7 \%)$ delas sofreram mais de um ataque por um mesmo forídeo ou por forídeo diferente. Duzentos e sessenta e duas formigas com pupa do parasitóide foram recuperadas do lixo. Além disso, pela dissecção estimou-se que mais dez operárias estivessem parasitadas porque tinham larvas mortas do parasitóide em suas cabeças. Deste modo, o número de formigas atacadas com formação de pupas ou somente larvas do parasitóide foi 272 , indicando parasitismo em $63,8 \%$ das formigas atacadas.

Houve emergência de 218 moscas, 114 machos $(52,3 \%)$ e 104 fêmeas $(47,7 \%$ ), o que indicou viabilidade pupal de $83,2 \%$ 
Tabela I. Comparações entre dois ninhos das variáveis obtidas pela liberação individual de fêmeas do forídeo N. elongata em uma cuba de observação com trânsito de operárias de A. sexdens rubropilosa.

\begin{tabular}{lcccc}
\hline \multicolumn{1}{c}{ Variável } & Ninho 1 $(\mathrm{n}=9)$ & Ninho 2 $(\mathrm{n}=15)$ & $\mathrm{t}$ & $\mathrm{P}$ \\
\hline Fluxo (número de formigas/3 min) & $64,8 \pm 13,3$ & $142,4 \pm 28,0$ & 8,34 & $<0,0001$ \\
Número de toques do forídeo nas formigas & $44,3 \pm 42,5$ & $83,9 \pm 38,7$ & 2,69 & 0,021 \\
Número de ataques do forídeo nas formigas & $11,2 \pm 7,2$ & $23,7 \pm 12,3$ & 2,02 & 0,062 \\
Relação número de toques/ataque & $4,9 \pm 3,9$ & $4,5 \pm 3,1$ & 0,07 & 0,940 \\
\hline
\end{tabular}

e razão sexual de 1,11 machos por fêmea. O tamanho das moscas fêmeas que emergiram em laboratório $(2,4 \pm 0,2 \mathrm{~mm})$ foi significativamente maior $(\mathrm{t}=4,44 ; \mathrm{P}=0,0001 ; \mathrm{g} .1 .=39)$ do que o tamanho dos machos $(2,2 \pm 0,1 \mathrm{~mm})$ e significativamente menor $(\mathrm{t}=3,70 ; \mathrm{P}=0,0005 ;$ g.l. $=51)$ do que o tamanho das fêmeas coletadas no campo $(2,7 \pm 0,3 \mathrm{~mm})$.

Cento e sete formigas atacadas morreram com menos de seis dias depois dos ataques, que foi o tempo mínimo desde a oviposição até a formação da pupa do parasitóide. A largura da cápsula cefálica de uma amostra destas formigas sem formação de pupa $(2,8 \pm 0,4 \mathrm{~mm})$ foi significativamente menor do que a largura da cápsula cefálica das formigas das quais houve emergência do parasitóide $(3,1 \pm 0,5 \mathrm{~mm})$ (Fig. 1). As formigas com pupa mas sem emergência de moscas apresentaram a largura da cápsula cefálica $(2,9 \pm 0,5 \mathrm{~mm})$ estatisticamente similar àquelas sem a formação de pupa e inferior àquelas em que houve emergência do parasitóide (Fig. 1). Em cerca de $40 \%$ das pupas do parasitóide das quais não emergiram moscas, foi constatada a ausência dos sifões respiratórios.

As moscas alimentadas com solução de mel 10\% tiveram maior longevidade do que as moscas submetidas aos outros tratamentos. A solução de mel $50 \%$ ou água destilada proporcionaram longevidade similar, que foi superior à longevidade das moscas em jejum (Fig. 2).

Dentre as fêmeas do parasitóide que emergiram em laboratório, 56 foram liberadas individualmente na cuba e, destas, 27 (48,2\%), com idade de um a quatro dias e sob as diversas condições de alimentação, iniciaram perseguição e investidas contra o hospedeiro, realizando ataques em pelo menos 200 formigas. As fêmeas demonstraram os mesmos comportamentos de voo e de ataque das fêmeas coletadas no campo, embora muitas delas não iniciassem perseguição às formigas tão logo fossem liberadas na cuba. No entanto, em nenhuma formiga daquelas colocadas na câmara ou removidas do lixo foi observada a presença de pupa do parasitóide. Não é possível afirmar se houve cópula dos casais mantidos nos tubos por 24 à $48 \mathrm{~h}$ antes das fêmeas serem liberadas na cuba. Houve aproximação entre alguns machos e fêmeas liberados na cuba, mas sem ocorrer cópula. Curiosamente, alguns machos dos casais liberados na cuba perseguiram as formigas da mesma forma que as fêmeas, mas sem tocá-las.

Em campo e laboratório, foi verificado que várias fêmeas de $N$. elongata sobrevoaram e aproximaram-se da cabeça de várias içás com ou sem asas, mas nenhum ataque ou um simples toque nestas formigas foi observado.

\section{DISCUSSÃO}

Os números de toques e de ataques foram proporcionais ao fluxo de cada ninho na cuba, mas não foram correlacionados com o fluxo das repetições em cada ninho. Este resultado é diferente de um estudo realizado em trilhas naturais, que mostrou números de toques e de ataques de Neodohrniphora sp. aumentando com o fluxo de operárias de $A$. sexdens rubropilosa (Tonhasca 1996). Porém, isso pode ser devido a diferenças no número de moscas sobre as trilhas naturais ou nos recursos transportados pelas operárias, pois as características do substrato vegetal podem afetar o tamanho médio das operárias e a intensidade do fluxo (Tonhasca \& Bragança 2000; Silva et al. 2007). Os números de toques de quatro a cinco vezes maiores do que os de ataques podem significar que as moscas necessitam testar pelo tato a atividade da operária e por pista química a característica saudável do hospedeiro para o desenvolvimento de suas progênies (Tonhasca 1996; Wuellner et al. 2002).

Não foi possível observar onde exatamente $N$. elongata introduz seu ovipositor na região posterodorsal da cabeça do hospedeiro porque os ataques foram muito rápidos, mas baseado em estudos com outras espécies o ovo deve ter sido introduzido pelo "forame magnum" da formiga (Tonhasca 1996; Feener \& Brown 1993). Neodohrniphora elongata e outros forídeos que atacam a cabeça das saúvas raramente ovipositam em formigas com carga porque, aparentemente, o fragmento vegetal impede a aproximação da mosca para ovipositar (Feener \& Brown 1993; Tonhasca 1996; Tonhasca et al. 2001), mas as espécies que atacam o abdome das operárias não discriminam entre formigas com e sem carga porque o fragmento vegetal não impede a oviposição (Bragança et al. 2002, 2003). Durante o ataque, $N$. elongata aparentemente deposita um único ovo no interior da cápsula cefálica da operária, assim como outros forídeos parasitóides de saúvas do mesmo gênero (Tonhasca 1996; Bragança et al. 2002).

A aglomeração das companheiras em torno das operárias recém-atacadas também foi observada em outros estudos com forídeos (Tonhasca 1996; Tonhasca et al. 2001), e supostamente representa um comportamento de "grooming" por causa da injúria provocada pela introdução do ovipositor da mosca ou porque ela deposita odores desconhecidos sobre a cutícula do hospedeiro atacado (Wuellner et al. 2002). Os hidrocarbonetos cuticulares são utilizados pelas formigas como sinais químicos de reconhecimento das companheiras de ninho (Whitehouse \& Jaffé 1995). 
A utilização do conteúdo da cápsula cefálica das operárias como alimento pelas larvas do parasitóide é comum no parasitismo de Atta spp. por forídeos de diversos gêneros, independentemente do local de oviposição no corpo da saúva (Bragança et al. 2002, 2003). Porém, a presença de uma pupa de $N$. elongata entre as mandíbulas de A. sexdens rubropilosa é um sinal comum somente de operárias parasitadas por forídeos do gênero Neodohrniphora (Tonhasca 1996; Bragança et al. 2002, 2003), mas diferente de operárias atacadas por forídeos dos gêneros Apocephalus e Myrmosicarius (Erthal \& Tonhasca 2000; Tonhasca et al. 2001).

A especialização sobre operárias de Atta spp. de diferentes tamanhos parece ser uma maneira de várias espécies de forídeos coexistirem sobre uma mesma espécie de hospedeiro (Bragança et al. 2003). Os ataques de fêmeas de $N$. elongata contra operárias em geral maiores do que o tamanho médio das forrageadoras da colônia é uma característica observada também em fêmeas de outras espécies de Neodohrniphora que atacam operárias de Atta spp. (Tonhasca 1996; Bragança et al. 2002), enquanto fêmeas do gênero Myrmosicarius, que são forídeos menores do que Neodohrniphora spp., atacam operárias de classe de tamanho inferior (Tonhasca et al. 2001). Em uma mesma classe de tamanho, as operárias grandes oferecem maiores possibilidades de desenvolvimento das fases iniciais do forídeo, pois forrageadoras maiores possuem maior quantidade de alimento do que operárias pequenas (Feener 1987). De fato, a largura média da cápsula cefálica das operárias onde as pupas de $N$. elongata apresentavam-se malogradas ou não se formaram foi inferior a 3,0 mm, que é a largura média da cápsula das operárias de onde houve emergência do parasitóide.

A impossibilidade de obtenção de $N$. elongata de segunda geração de laboratório parece ser um importante aspecto que limita a produção de forídeos parasitóides de saúvas além da primeira geração, o que pode depender da obtenção de acasalamentos e estar relacionado à alimentação dos adultos de primeira geração. Apesar das fêmeas de laboratório terem realizado o comportamento de ataque semelhante às fêmeas de campo, não se pode afirmar que houve oviposição.

As fêmeas de espécies de forídeos do gênero Pseudacteon são aparentemente atraídas para as proximidades das colônias dos seus hospedeiros, as formigas Solenopsis spp., por sinais olfativos das formigas (Feener \& Brown 1992) e o acasalamento ocorre no local (Feener 1987). Grande número de machos de Pseudacteon spp. são atraídos até o ninho de Solenopsis saevissima em pré-enxameamento (antes e durante a revoada), sugerindo que machos provavelmente usam o ninho prérevoada como um sinal para encontrar as fêmeas que estão realizando ataques (Pesquero et al. 1993). A simulação destas condições naturais em laboratório resultou em acasalamentos de Pseudacteon spp. (Porter et al. 1997; Morrison et al. 1997). A perseguição das operárias pelos machos de $N$. elongata liberados na cuba parece indicar o comportamento de utilização da trilha de operárias para localizar a fêmea para acasalamento. Algumas vezes, durante a coleta de fêmeas de $N$. elongata, o acasalamento em Neodohrniphora spp. foi observado em pleno voo sobre trilhas ativas de A. sexdens rubropilosa e talvez a ocorrência deste comportamento de $N$. elongata em laboratório depende ainda do aprimoramento da simulação das condições de campo, como espaço maior para o voo de machos e fêmeas.

Os forídeos sobreviveram mais quando alimentados com solução de mel 10\%, mas para a multiplicação de forídeos de saúvas em laboratório é necessário conseguir que os indivíduos copulem e resolver o problema da aparente falta de oviposição das fêmeas que emergem em laboratório, o que pode depender de uma fonte protéica específica (Disney 1990). Nada se conhece acerca do que os adultos de forídeos parasitóides de formigas cortadeiras fazem quando eles não estão atacando as formigas ou do que eles se alimentam no campo. Por outro lado, estudos mostraram que soluções açucaradas ("honeydew") parece ser a fonte primária de alimentação da maioria dos forídeos adultos, os quais também podem ser predadores de insetos, se alimentarem de néctar de flores ou sugar a seiva de plantas (Disney 1994). Semelhantemente ao que está sendo feito de modo satisfatório para forídeos parasitóides do gênero Pseudacteon (Fadamiro \& Chen 2005; Chen \& Fadamiro 2006), estudos devem ser realizados para investigar a adequação de néctar e honeydew de diferentes fontes naturais sobre a longevidade e reprodução dos forídeos parasitóides de saúvas.

$\mathrm{O}$ parasitismo de fêmeas reprodutivas de formigas por forídeos é raro, sendo que somente um exemplo de parasitismo de um forídeo não identificado em Solenopsis invicta foi registrado (Wojcik et al. 1987). Tentativas de confirmar o parasitismo de fêmeas reprodutivas de Solenopsis spp. por Pseudacteon spp. não tiveram sucesso (Willians \& Banks 1987; Pesquero et al. 1993). As fêmeas reprodutivas de A. sexdens e outras espécies do gênero possuem as cápsulas cefálicas maiores do que as forrageadoras e, portanto, representam uma fonte de alimento potencialmente maior. Porém, a impossibilidade de ataque de $N$. elongata em içás de $A$. sexdens rubropilosa se justificaria porque elas são restritas ao período de revoada e ficam no solo expostas às moscas por curto período. Há necessidade de realizar estudos com outros forídeos que possam atacar içás porque a confirmação de parasitismo desta casta seria de grande valor para as perspectivas de contribuição destes parasitóides no manejo de saúvas.

Agradecimentos. A Deborah Coracini, Denise Moreira e Milton Erthal Jr. pela ajuda nos trabalhos de campo e laboratório, a Manoel J. Ferreira pelo auxílio na manutenção dos ninhos em laboratório e a Brian V. Brown pela identificação do forídeo. Ao CNPq pela concessão de bolsas de pesquisador. Esta pesquisa foi financiada por International Foundation for Science (IFS).

\section{REFERÊNCIAS}

Antunes, E. C.; R. N. C. Guedes; T. M. C. Della-Lucia \& J. E. Serrão. 2000. Sub-lethal effects of abamectin suppressing colonies of the leaf-cutting ant Acromyrmex subterraneus subterraneus. Pest Management Science 56: 1059-1064.

Antunes, E. C.; T. M. C. Della-Lucia; R. N. C. Guedes \& J. E. Serrão. 
2005. Abamectrin-driven alternations on queen ovaries of the leafcutting ant Acromyrmex subterraneus subterraneus (Hymenoptera, Formicidae). Sociobiology 45: 163-172.

Borgmeier, T. 1928. Nota prévia sobre alguns phorideos que parasitam formigas cortadeiras dos gêneros Atta e Acromyrmex. Boletim Biologico 14: 119-126.

Borgmeier, T. 1931. Sobre alguns phorideos que parasitam a saúva e outras formigas cortadeiras (Diptera: Phoridae). Archivos do Instituto Biologico 4: 209-228.

Bragança, M. A. L.; A. Tonhasca Jr. \& T. M. C Della Lucia. 1998. Reduction in the foraging activity of the leaf-cutting ant Atta sexdens caused by the phorid Neodohrniphora sp. Entomologia Experimentalis et Applicata 89: 305-311.

Bragança, M. A. L.; A. Tonhasca Jr. \& D. D. O. Moreira. 2002. Parasitism characteristics of two phorid fly species in relation to their host, the leaf-cutting ant Atta laevigata (Smith) (Hymenoptera: Formicidae). Neotropical Entomology 31: 241244.

Bragança, M. A. L.; T. M. C. Della Lucia \& A. Tonhasca Jr. 2003. First record of phorid parasitoids (Diptera: Phoridae) of the leaf-cutting ant Atta bisphaerica Forel (Hymenoptera: Formicidae). Neotropical Entomology 32: 169-171.

Bragança, M. A. L. \& Z. C. S. Medeiros. 2006. Ocorrência e características biológicas de forídeos parasitóides (Díptera: Phoridae) da saúva Atta laevigata (Smith) (Hymenoptera: Formicidae) em Porto Nacional, TO. Neotropical Entomology 35: $408-411$.

Brown, B. V. 2001. Taxonomic revision of Neodohrniphora, subgenus Eibesfeldtphora (Diptera: Phoridae). Insect Systematics and Evolution 32: 393-409.

Chen, L. \& H. Y. Fadamiro. 2006. Comparing the effects of five naturally occurring monosaccharide and oligosaccharide sugars on longevity and carbohydrate nutrient levels of a parasitic phorid fly, Pseudacteon tricuspis. Physiological Entomology 31: 4656.

Cherrett, J. M. 1986. The biology, pest status and control of leafcutting ants. Agricultural Zoology Reviews 1: 1-37.

Disney, R. H. L. 1990. Some myths and the reality of scuttle fly biology. Antenna 14: 64-67.

Disney, R. H. L. 1994. Scuttle flies: the Phoridae. London, Chapman \& Hall, $467 \mathrm{p}$.

Disney, R. H. L. \& M. A. L. Bragança. 2000. Two new species of Phoridae (Diptera) associated with leaf-cutter ants (Hymenoptera: Formicidae). Sociobiology 36: 33-39.

Erthal, M. Jr. \& A. Tonhasca Jr. 2000. Biology and oviposition behavior or the phorid Apocephalus attophilus and the response of its host, the leaf-cutting ant Atta laevigata. Entomologia Experimentalis et Applicata 95: 71-75.

Fadamiro, H. Y. \& L. Chen. 2005. Utilization of aphid honeydew and floral néctar by Pseudaction tricuspis (Diptera: Phoridae), a parasitoid of imported fire ants, Solenopsis spp. (Hymenoptera: Formicidae). Biological Control 34: 73-82.

Feener, D. H. Jr. 1987. Size-selective oviposition in Pseudacteon crawfordi (Diptera: Phoridae), a parasite of fire ants. Annals of the Entomological Society of America 80: 148-151.

Feener, D. H. Jr. \& B. V. Brown. 1992. Reduced foraging of Solenopsis geminata (Hymenoptera: Formicidae) in the presence of parasitic Pseudacteon (Diptera: Phoridae). Annals of the Entomological Society of America 85: 80-84.

Feener, D. H. Jr. \& B. V. Brown. 1993. Oviposition behavior of an antparasitizing fly, Neodohrniphora curvinervis (Diptera: Phoridae), and defense behavior by its leaf-cutting ant host Atta cephalotes (Hymenoptera: Formicidae). Journal of Insect Behavior 6: 675688.

Feener, D. H. Jr. \& K. A. G. Moss. 1990. Defense against parasites by hitchhikers in leaf-cutting ants: a quantitative assessment.
Behavioral Ecology and Sociobiology 26: 17-29.

Fowler, H. G.; M. I. Pagani; O. A. Silva; L. C. Forti; V. Pereira-da-Silva \& H. L. Vasconcelos. 1989. A pest is a pest is a pest? The dilemma of Neotropical leaf-cutting ants: keystone taxa of natural ecosystems. Environmental Management 13: 671-675.

Morrison, L. W. \& S. D. Porter. 2005. Testing for population-level impacts of introduced Pseudacteon tricuspis flies, phorid parasitoids of Solenopsis invicta fire ants. Biological Control 33: 9-19.

Morrison, L. W.; C. G. Dall'Aglio-Holvorcem \& L. E. Gilbert. 1997. Oviposition behavior and development of Pseudacteon flies (Diptera: Phoridae), parasitoids of Solenopsis fire ants (Hymenoptera: Formicidae). Environmental Entomology 26: $716-724$.

Orr, M. R. 1992. Parasitic flies (Diptera: Phoridae) influence foraging rhythms and caste division of labor in the leaf-cutter ant, Atta cephalotes (Hymenoptera: Formicidae). Behavioral Ecology and Sociobiology 30: 395-402.

Pesquero, M. A.; S. Campiolo \& H. G. Fowler. 1993. Phorids (Diptera: Phoridae) associated with mating swarms of Solenopsis saevissima (Hymenoptera: Formicidae). Florida Entomologist 76: 179-181.

Porter, S. D.; D. E. Williams \& R. S. Patterson. 1997. Rearing the decapitating fly Pseudacteon tricuspis (Diptera: Phoridae) in imported fire ants (Hymenoptera: Formicidae) from the United States. Journal of Economic Entomology 90: 135-138.

Silva, V. S. G.; O. Bailez; A. M. Viana-Bailez \& A. Tonhasca Jr. 2007. Effect of the size of workers of Atta sexdens rubropilosa on the attack behavior of Neodohrniphora spp. (Diptera: Phoridae). Sociobiology 50: 35-44.

Tonhasca, A. Jr. 1996. Interactions between a parasitic fly, Neodohrniphora declinata (Diptera: Phoridae), and its host, the leaf-cutting ant Atta sexdens rubropilosa (Hymenoptera: Formicidae). Ecotropica 2: 157-164.

Tonhasca, A. Jr. \& M. A. L. Bragança. 2000. Effect of leaf toughness on the susceptibility of the leaf-cutting ant Atta sexdens to attacks of a phorid parasitoid. Insectes Sociaux 47: 220-222.

Tonhasca, A. Jr.; M. A. L. Bragança \& M. Erthal Jr. 2001. Parasitism and biology of Myrmosicarius grandicornis (Diptera, Phoridae) in relationship to its host, the leaf-cutting ant Atta sexdens (Hymenoptera, Formicidae). Insectes Sociaux 48: 154-158.

Vazquez, R. J.; S. D. Porter \& J. A. Briano. 2006. Field release and establishment of the decapitating fly Pseudacteon curvatus on red imported fire ants in Florida. BioControl 51: 207-216.

Weber, N. A. 1972. Gardening ants, the Attines. Memoirs of the American Philosophical Society 92: 1-146.

Whitehouse, M. E. A. \& K. Jaffé. 1995. Nest mate recognition in the leaf-cutting ant Atta laevigata. Insectes Sociaux 42: 157-166.

Williams, D. F. \& W. A. Banks. 1987. Pseudacteon obtusos (Diptera: Phoridae) attacking Solenopsis invicta (Hymenoptera: Formicidae) in Bazil. Psyche 94: 9-13.

Wilson, E. O. 1980. Caste and division of labor in leaf-cutter ants (Hymenoptera: Formicidae: Atta) - I. The overall pattern in A. sexdens. Behavioral Ecology and Sociobiology 7: 143-156.

Wojcik, D. P.; D. P. Jouvenaz \& C. S. Lofgren. 1987. First report of a parasitic fly (Diptera: Phoridae) from a red imported fire ant (Solenopsis invicta) alate female (Hymenoptera: Formicidae). Florida Entomologist 70: 181-182.

Wuellner, C. T.; C. G. Dall'Aglio-Holvorcen; W. W. Benson \& L. E. Gilbert. 2002. Phorid fly (Diptera: Phoridae) oviposition behavior and fire ant (Hymenoptera: Formicidae) reaction to attack differ according to phorid species. Annals of the Entomological Society of America 95: 257-266.

Zanetti, R.; E. F. Vilela; J. C. Zanuncio; H. G. Leite \& G. D. Freitas. 2000. Influência da espécie cultivada e da vegetação nativa circundante na densidade de sauveiros em eucaliptais. Pesquisa Agropecuária Brasileira 35: 1911-1918.

Recebido em 26/02/2008; aceito em 15/10/2009 